THE central theme of the symposium was structure at the molecular level. Many fibrous proteins have a highly regular structure; for example, the collagen of tendon shows nature adopting at the molecular level the principle of successive twisting that man has developed in the rope. Man has not yet developed a molecular rope, but different methods of drawing polyethylene lead to fibres with a wide variety of mechanical properties, some with the stiffness of glass and the tensile strength of steel (Ward, Leeds). The mechanical properties may be related to the structural regularity of the molecular folds in the polymer. There is a striking similarity between the folded chains of polyethylene (Sadler, Bristol) and those of the protein in the egg stalk of the lacewing fly, chrysopa, but many natural polymers share with the synthetics an intermingling of regular and less regular structural regions. In the globular enzymes, the same types of chain conformation are found as in fibrous proteins, but each stretch is limited in extent so that the chain can fold to form a compact shape (North, Leeds). The recurrence of certain well defined folds in different enzymes probably indicates evolution from a common ancestral protein, though there remains the possibility that some conformations may recur just because they are energetically favourable, as do the folds in polyethylene.

The alternation of regularity with irregularity is seen too in polysaccharides, the formation of gels of which depends upon molecular chains being associated in pairs for limited distances (Rees, Unilever); interruption of the regular alternating copolymer by a sugar unit with a different conformation makes a kink in the chain, causing the molecular partnership to break and the two chains to diverge before joining up with different partners. The variety of sugar units utilised in polysaccharides permits a great range of conformations, some of which are very susceptible to changes in solvent environment (Atkins, Bristol); thus conformational changes similar to those induced in heparan sulphate by calcium may be a vital factor in cell adhesion.

In the chromatin of the cell nucleus, the nucleic acid is intimately associated with histones, basic proteins of five types. It is now beginning to appear that the histone molecules are individually globular and associate specifically to form a bead-necklace around which the nucleic acid is wound (Thomas, Cambridge).
A second theme of the conference was chemical modification of polymers. Technologists may attempt to modify a polymer in order to improve its properties, such as the drape and

\section{Natural and synthetic polymers}

With the praiseworthy intent of allowing enzymologists and polymer scientists to learn from each other, the SRC Enzyme Chemistry and Technology Committee organised a meeting on January 2 and 3 at Cambridge. In three sessions the meeting dealt with synthetic polymers, structural natural polymers and enzymes. The reports of the meeting which follow describe it from the point of view of, respectively, an enzymologist and a polymer scientist.

crease-resistance of cotton fabrics (Roberts, Shirley Institute). Protein workers often use chemical modification to a different end, in probing structure and function. Very high degrees of specificity may be achieved (Dixon, Cambridge), though care must be exercised in drawing conclusions from the effects of such modifications - the activity of an enzyme may be perturbed by changes remote from its active site. Such methods have been applied elegantly in distinguishing the part played by individual subunits in a multimeric enzyme and in studying the interactions between components of a multi-enzyme complex and between the subunits of a virus (Perham, Cambridge).

The Cambridge meeting established interesting overlaps between the work on natural and synthetic fibres, for example in the molecular basis of mechanical properties and in the thermodynamic and kinetic constraints on the folding pathways. While the programme had a bias towards the natural fibres, showing clearly the great subtlety, elegance and efficiency with which nature has evolved a wide variety of functions from a limited repertoire of structural elements, it is clear that man has already achieved enormous progress in a very short time in making an almost comparable variety of synthetic materials.
Experiments 'with model compounds (Kirby, Cambridge) show how chemical reaction rates depend critically upon the reacting groups being constrained into an appropriate geometry; such studies are vital to the eagerly awaited development of catalysts with the efficiency and specificity of enzymes-which will doubtless owe much to comparative studies of natural and synthetic polymers.

A. C. T. NORTh

Scientists have great trouble even in communicating the meaning of their work to each other. This was probably the main conclusion to be drawn from the symposium which, because of the lack of dialogue or (apparently) of flashes of inspiration, must be judged a failure. The reasons may be that the speakers did not address themselves sufficiently to areas of potential overlap, that the discussion was too limited or that the two subjects are irrelevant to each other.

Polymer science groups in the USA are increasingly turning their interest to biological macromolecules, spurred on by the increase in the fortunes of the NIH compared to those of the Defense Department a few years ago. This has resulted in work on solid state and solution properties of polysaccharides and polypeptides, one obvious avenue, and in the study of collagen ultrastructure and its relationship to mechanical properties presented by R. G. C. Arridge and L. Gathercole of Bristol and carried out jointly with Case Western Reserve University.

One thing that did emerge was the relative lack of understanding of physical aspects of enzyme structure such as the denaturation and renaturation processes, the nature of subunit interactions, and of questions such as "How large must a protein be to have a hydrophobic core?" posed by T. L. Blundell during the discussion. This was reinforced by the parallel between J. O. Thomas's review of work on chromatin structure at Cambridge and D. M. Sadler's of polymer crystallisation and morphology. Both topics are almost baroque in their complexity, the methods used are completely different, yet the problems are very much related. It is to be hoped that researchers starting from the synthetic polymer direction can contribute to these subjects, though the immediate problem for a person used to working with kilograms of sample is to learn techniques for handling natural polymers, and here cooperation between the groups is essential.

P. D. Calvert 